\title{
The Mechanism of Carthamin Yellow Injection Promote Acute Myocardial Infarction Infarct Border Zone Angiogenesis Xiao-min WANG $^{1}$, Zhi-yu ZHOU ${ }^{1}$, Yao-kun XIONG ${ }^{1, *}$, Jiang-tao $\mathrm{WEI}^{2}$, Yan-ting WAN ${ }^{3}$, Fei $\mathrm{LI}^{1}$ and Zhi-yong $\mathrm{LIU}^{1, *}$ ${ }^{1}$ Jiangxi University of Traditional Chinese Medicine, Nanchang 330004, China. \\ ${ }^{2}$ Department of Cadre ward, the fourth Affiliated Hospital of Nanchang University, Nanchang 330002,China. \\ 3 Jiangxi People's Armed Police Corps Hospital, Nanchang 330001,China. 56264931@qq.com(Y.K. Xiong), 469241547@qq.com(Z.Y. Liu). \\ ${ }^{*}$ Corresponding authors.
}

Keywords: Carthamin Yellow, Vascular Regeneration, Vascular Endothelial Growth Factor, Acute Myocardial linfarction.

\begin{abstract}
We observed the impact of carthamin yellow injection on revascularization in rats with acute myocardial infarction and discuss its mechanism. The left anterior descending branches of the coronary arteries of Wistar rats were ligatured, and the animal model with acute myocardial infarction was duplicated. The animals with successful modeling were randomly divided into the model group and treatment group. The rats in the treatment group were injected with carthamin yellow $(2.5 \mathrm{mg} / \mathrm{Kg} \bullet \mathrm{d})$ for 3, 7 and 14 days. Their weights were measured $24 \mathrm{~h}$ after the last drug administration, and their hearts were removed for weighing and blood collection. The Cardiac indices were then measured, and electrocardiograms (ECGs) were recorded. Masson staining was used to determine the microvessel count of the infarct border zone of the myocardium. Immunohistochemistry was used to detect the expression of vascular endothelial growth factor (VEGF). Compared with the model group, the cardiac indices of the rats treated with carthamin yellow for 7 and 14 days were decreased $(\mathrm{P}<0.05)$. The ECGs of these treated rats returned to normal. In the drug administration groups, the number of capillaries at the marginal zone of the myocardial infarction clearly increased $(\mathrm{P}<0.05)$. The immunohistochemical results showed that the expressions of VEGF at the marginal zone of acute myocardial infarction increased $(\mathrm{P}<0.05)$. The injection of carthamin yellow has therapeutical effects on rats with acute myocardial infarction. It can promote the revascularization of the myocardium at the marginal zone of myocardial infarction. This effect could be related to the increase in VEGF expression at the marginal zone of acute myocardial infarction.
\end{abstract}

\section{Introduction}

Carthamin yellow is one of the main active ingredients of traditional Chinese medicine (TCM) red flowers, including Safflower yellow pigments $\beta$ and $\alpha$. Carthamin yellow consists of many chalcone compounds and can be found in the water-soluble extract of safflower [1]. Carthamin yellow is known to increase coronary blood flow and improve myocardial ischemia and myocardial damage $[2,3]$. In many patients with microcirculation blocking due to myocardial infarction, the heart cannot effectively perfuse. A variety of vascular growth factor and its effect on the gene transfer treatment to promote angiogenesis has been approved, but in clinical 
applications are still some problems such as effectiveness and safety. This study investigates the effect of safflower yellow on the infarct edge vascular regeneration of rats with acute myocardial infarction along with the mechanism of the effect.

\section{Materials and Methods}

\section{Experime ntal Materials}

Experimental Animals. The rats used were healthy adult Sprague-Dawley (SD) rats (male, clean level, weight $=200 \pm 10 \mathrm{~g}$ ) purchased from Hunan Weasley, Laboratory Animal Co., Ltd. (license SCXK. 2012-0004). Those animals feeding based hospital laboratory animal center in Jiangxi College of Traditional Chinese Medicine (demonstration teaching center in the Jiangxi Province Department of Education). Animals are in adaptive feed start a week after the experiment. All the animals were kept together in one room under the same breeding environment and conditions. The experimental rats were given free access to food and water. The room temperature was $20-22^{\circ} \mathrm{C}$, and the humidity ranged from $45 \%$ to $65 \%$. The light-dark cycle was 12 hours of light and 12 hours of darkness.

Experimental Drug. Safflower yellow pigment (Zhejiang Yongning Pharmaceutical Co., Ltd.) was injected as the experimental drug.

Reagents and Instrumentation. Mason dyeing liquid (Shanghai Bioengineering Co., Ltd); a fight and VEGF DAB chromogenic box (Beijing Chinese fir biological reagents, batch number K133319D); a physiological signal acquisition system (Invite Raw Technology Co., Ltd., Sichuan); a YB - 6 d biological tissue paraffin embedding machine and a YT - 6 c type biological tissue stand and baking machine (Hubei Xiaogan Inferior Smooth Medical Electronic Technology Co., Ltd.); a type PH140A incubator/drying box; a horizontal Scientific Instrument Co., Ltd. (Shanghai); and a Leica DL2500 microscope and CCD image acquisition system (Leica, Germany).

\section{Experime ntal Methods}

AMI Model.[4] Adaptive Wistar rats fed three days later, make body Jones lang lasalle ECG monitoring after weighing and anesthesia fixed in the table on your back. Conventional iodine volts after disinfection and sternum left edge rib between 3-4 skin incision, step by step a blunt separation subcutaneous tissue, muscle, left hand gently press right chest out of the he art, with 0 the curved needle thread ligation of coronary artery from the origin of left coronary artery 2-3 $\mathrm{mm}$. Ligation place the following myocardial white, heart beat is abate, and lead electrocardiogram II ST broken arch upward obviously improved for building successful symbol. Then use 10 $\mathrm{ml}$ syringe get chest excess air to produce negative pressure, closing chest, step by step a partial to penicillin powder to prevent infection.

Grouping Method. After three days of feeding, the experimental animals were randomly divided into two safflower yellow injection treatment groups (either 7 or 14 days of treatment) and a model group. Each group contained 10 rats.

Dosing Method. Safflower injection group was given intraperitoneal injection of ( $2.5 \mathrm{mg} / \mathrm{Kg}$ ), respectively, for 3 days, 7 days, 14 days, the model group was given equal normal saline. administered at a concentration of $1.25 \mathrm{mg} / \mathrm{mL}$ via a normal saline injection. 


\section{Measure ments}

\section{Heart Index Detection}

After fasting for $12 \mathrm{~h}$, each animal was weighed. After anesthesia was administered, the heart was opened under aseptic conditions and washed with physiological saline. Sterile gauze was used to absorb surface moisture, and the cardiac weight index was calculated.

\section{Microvascular Count}

An image acquisition system was used to collect images of the edge area of infarction. Low magnification $(10 \times 10)$ was used to scan the entire section. Subsequently, we identified five "hot spots" with the highest densities of blood vessels around the edge of the infarction. These spots were then reimaged at high magnification $(10 \times 40)$. Each slice observation random, middle, bottom, left and right ten high field of vision, count each view of microvascular number take its combined.

\section{Immunohistochemical Analysis of VEGF Protein Expression.}

Tissue specimens of normal paraffin embedding, sectioning, thickness of about 2 microns. Dewaxing, hydration by $3 \% \mathrm{H}_{2} \mathrm{O}_{2}$ after incubation for $10 \mathrm{~min}$ at room temperature, eliminate endogenous peroxidase activity, hot fix antigen $5 \mathrm{~min}$. Add antibody of VEGF (10) $37^{\circ} \mathrm{C}, 2 \mathrm{~h}$ incubation, DAB chromogenic at room temperature. Wood grain redyeing, dehydration, transparent, neutral gum sealing piece. And set up positive control and negative control, with a known positive section as the positive control; With PBS instead of a fight, as the negative control. VEGF protein expression in the cytoplasm, present different degree of yellow or brown granules, each section to determine 400 times 5 field of vision, the Image - Pro Plus 6.0 Image analysis system (IPP) computing positive region the average optical density of the area of the $\mathrm{IOD} /$ positive area ratio for the positive expression of strength.

\section{Experime ntal Results}

\section{Influence of Central Index}

In the treatment group, the heart index declined gradually compared to the model group. After three days of treatment, no statistical difference was observed $(\mathrm{P}>0.05)$. In contrast, the differences in heart index were significant after $7(\mathrm{P}<0.01)$ and 14 days $(\mathrm{P}<0.01)$ of treatment (Table 1$)$.

Table 1 Effects of safflower yellow on the heart weight indices of rats with acute myocardial infarction

$$
(\bar{x} \pm s)
$$

\begin{tabular}{lll}
\hline Group & Number of rats & Heart index $(\mathrm{g} / 100 \mathrm{~g})$ \\
\hline Model & 6 & $0.36 \pm 0.09$ \\
Treatment (3 days) & 6 & $0.37 \pm 0.01$ \\
Treatment (7 days) & 6 & $0.32 \pm 0.01^{*}$ \\
Treat ment (14 days) & 6 & $0.29 \pm 0.06^{*}$ \\
* Compared with model group: P $<0.05$ &
\end{tabular}




\section{Impacts on ECGs}

All the duration of treatment increased from three to 14 days, the ST segment of the ECG gradually declined. After two minutes of model group electrocardiogram shows Rsr 'type, 14 days in qRs type. 3 days group rats electrocardiogram (ECG) changes: 2 minutes after the Rsr 'type, three days later a QR, ST segment elevation. Set of ecg changes in rats: 7 days after 2 minutes in qRs type, ecg returned to normal in 7 days. Set of ecg changes in rats: 14 days after 2 minutes of ST segment is a carpenter raise up, after 14 days ecg returned to normal.

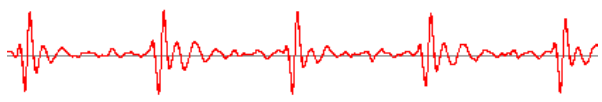

odel group after 2 minutes

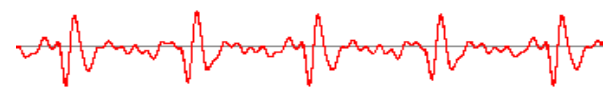

Treat ment group (3 days) after 2 minutes

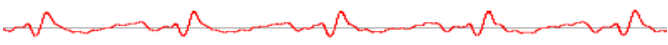

Treat ment group (7 days) after 2 minutes

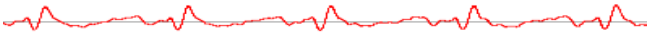

Treat ment group (14 days) after 2 minutes

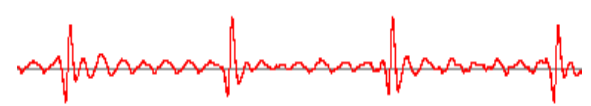

Put to death before the model group

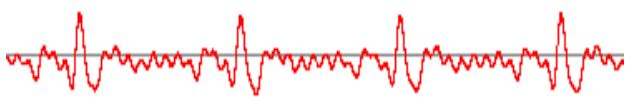

Treatment group (3 days)before death

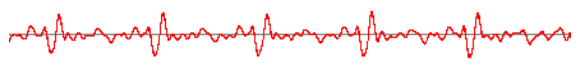

Treat ment group (7 days) before death

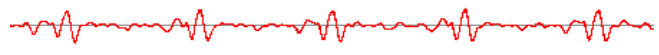

Treatment group (14 days)before death

Figure 1 Electrocardiogram of postoperative and sacrificed on myocardial infarction rats

\section{Effects on Microvascular Generation}

In the model group, no obvious increase in the microvascular density was observed in the infarction edge area. In the treatment group, an obvious increase in the number of capillaries was observed in the infarction edge area. Compared with the model group, the differences in capillary number were significant after all treatment durations $(\mathrm{P}<$ 0.05; Table 2 and Figure 2).

Table 2 Effects of safflower yellow on the mic rovascular densities of rats with acute myocardial infarction $(\bar{x} \pm s)$

\begin{tabular}{lcc}
\hline Group & Nu mber of rats & Microvascular density \\
\hline Model & 6 & $519 \pm 121$ \\
3 days & 6 & $748 \pm 249^{*}$ \\
7 days & 6 & $753 \pm 231^{*}$ \\
14 davs & 6 & $1062 \pm 214^{* *}$ \\
\hline
\end{tabular}

*Compared with model group*: $\mathrm{P}<0.05 ; * *$ Co mpared with model group: $\mathrm{P}<0.01$ 


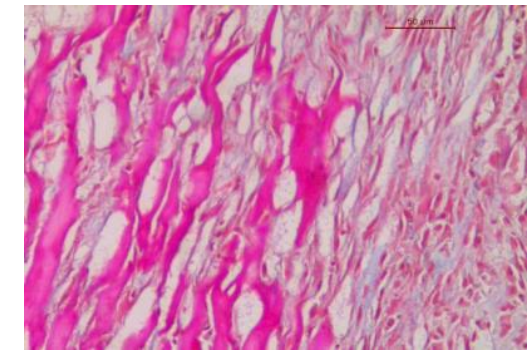

Model group (10 x 20)

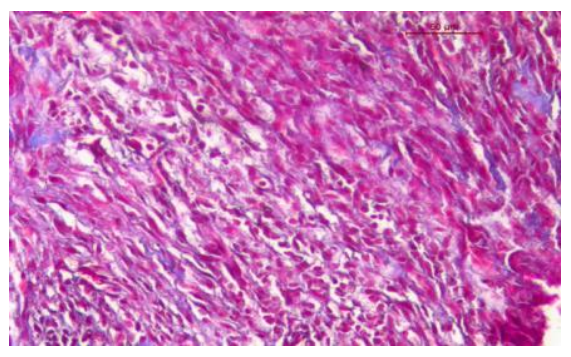

Treat ment group 7 days $(10 \times 20)$

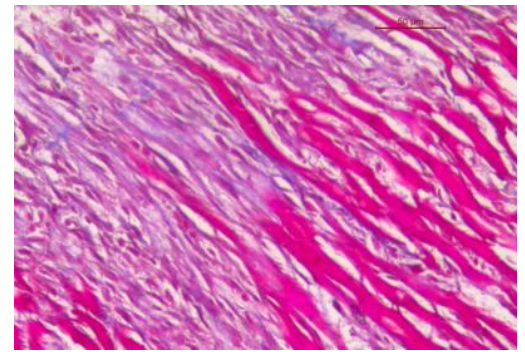

Treat ment group 3 days $(10 \times 20)$

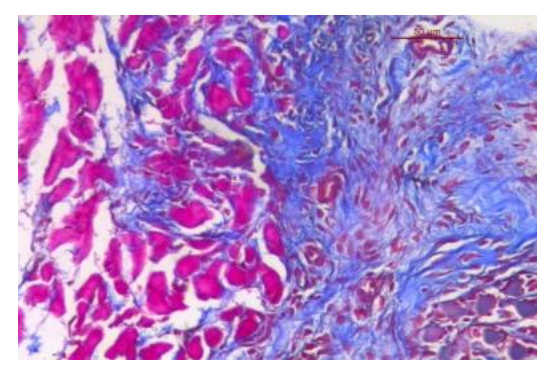

Treat ment group 14 days $(10 \times 20)$

Figure 2 Mass on staining of myocardial infarction rats

\section{Effects on VEGF Expression}

In the treatment group, the VEGF expression increased gradually in the edge area of myocardial infarction with increasing treatment duration. Compared with the model group, the VEGF expression increased after treatment for three and seven days, although the differences were not significant $(\mathrm{P}>0.05)$. After 14 days of treatment, the difference in VEGF expression was statistically significant $(\mathrm{P}<0.05$; Table 3 and Figure 3).

Table 3 Effects of safflower yellow on the VEGF expression of rats with acute myocardial infarction

$$
(\bar{x} \pm s)
$$

\begin{tabular}{lll}
\hline Group & Nu mber of rats & Infarction area \\
\hline Model & 6 & $0.21 \pm 0.01$ \\
3 days & 6 & $0.22 \pm 0.01$ \\
7 days & 6 & $0.25 \pm 0.03$ \\
14 days & 6 & $0.33 \pm 0.08^{*}$ \\
\hline
\end{tabular}

*Compared with model group: $\mathrm{P}<0.05$ 


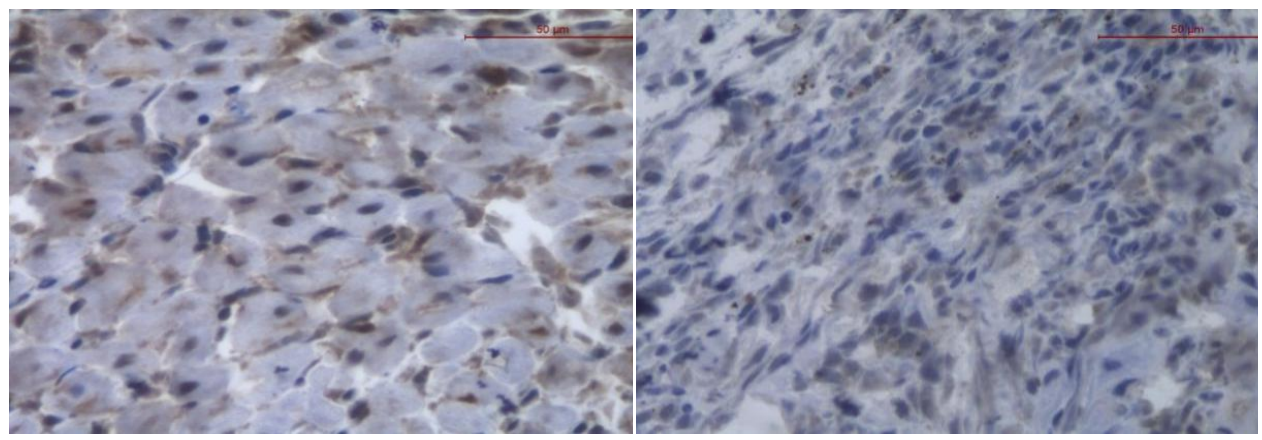

Model group (10 x 20)

Treatment group 3 days $(10 \times 20)$

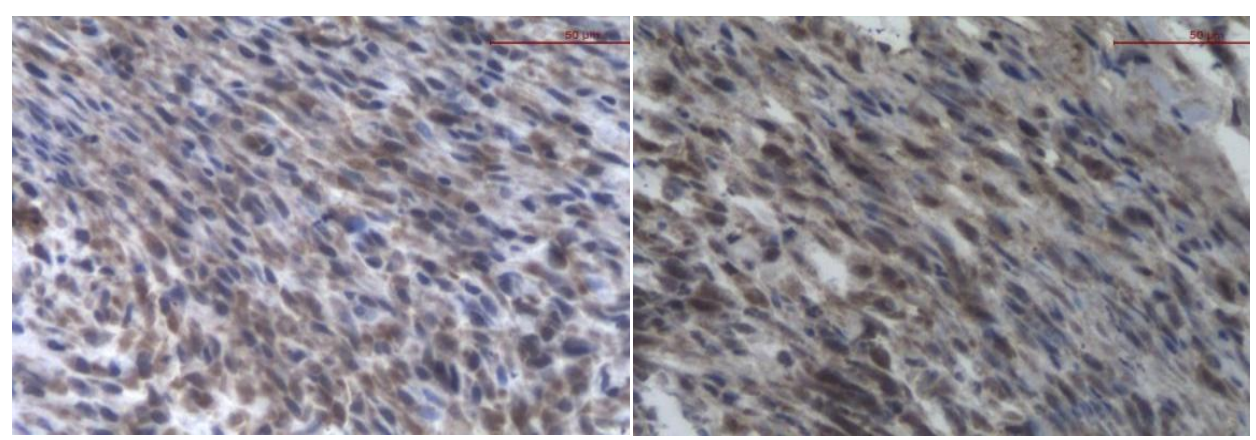

Treat ment group 7 days $(10 \times 20)$

Treat ment group 14 days $(10 \times 20)$

Figure 3. Immunohistochemical was used to measure the protein expression of VEGF

\section{Discussion}

Currently, the treatment for acute myocardial infarction associated with coronary artery disease mainly includes drug therapy after coronary interventional therapy and surgery. However, for serious acute myocardial infarctions resulting in pathological changes in the coronary artery, the effectiveness of traditional medications is limited. and the coronary artery interventional therapy and bypass grafting were $30 \%-30 \%$ of the rate of restenosis, and part of the patient's individual situation does not adapt to surgery [5]. In view of the above treatment limitations, the improvement of microcirculation has become a focus of efforts to promote the angiogenesis of areas of acute myocardial infarction [6].

In this study, rats with myocardial infarction were treated by the injection of safflower yellow followed by dynamic observation of ECGs and observation of stained blood vessels in the infarction edge area. This results indicate that after three and seven days of treatment with safflower yellow, the cardiac index and ECG recovery were not significantly different from the model group. After 14 days of treatment, the ST segment of the rat ECG was close to normal. The Masson staining results indicate that the injection of safflower yellow pigment had a regenerative effect on the blood vessels in the infarction edge area.

VEGF is the body's strongest angiogenesis-promoting factor. It promotes vascular endothelial cell mitosis and migration along with endothelial cell proliferation and prompts intravascular proteins to leave the cell to build blood vessels; VEGF also supports endothelial cells and fibroblasts $[6,7]$, the migration of fibroblast synthesis, and secretion of matrix protein and proteoglycan, and endothelial cells to form new blood vessels, and increase microvascular permeability, is a key in the process of the formation of blood vessels of regulating factor $[8,9]$. A considerable amount of basic 
research and clinical trials have confirmed that VEGF protein or gene therapy is an effective treatment for ischemic heart disease. Will human recombinant VEGF protein or VEGF genes into the animal model of ischemic myocardium can promote angiogenesis and improve heart function [10]. The experimental results show that after 14 days of treatment with safflower yellow pigment, VEGF expression increased significantly at the infarction edge area. In addition, the vascular density also increased in this area. This is clearly associated with the expression of VEGF, as VEGF promotes endothelial cell growth and proliferation. Safflower yellow pigment is an important part of the red flower of TCM for activating blood circulation, and to have new, remove stasis blood detumescence can also raw muscle. Its possible mechanism is to mobilize the safflower yellow pigment body parts of endothelial progenitor cells migrated to ischemia, through direct participation and factor secreted into the blood vessels, promote new blood vessels to form. However, the specific mechanism requires further in-depth study.

\section{Acknowledgme nts}

This research was financially supported by Jiangxi province natural science foundation of China (2012BAB205096) , (2014ZBBG70012) and Jiangxi provincial health department foundation of China (2014A022), (2014A096). We are pleased to thank F.Y. XIAO for the English language polishing and F. Li for her excellent assistance in editorial changes.

\section{References}

[1] Zhifu Y, Qibing M, Yongpei J, et al. Safflower active ingredients and pharmacological effects. Pharmaceutical journal of northwest.16(2001) 131-133.

[2] Wanqun $X$, Lei S, Huali G, et al. Safflower yellow pigment can affect inflammatory factor for acute coronary syndrome patients. Journal of Qingdao university medical college. 47(2011)426-428.

[3] Zhang Q, Zheng DY, Xu HY, etc. Safflower yellow pigment on focal cerebral ischemia-reperfusion injury in rats of protection. Journal of shandong university (medical), 49(2011)11-14.

[4] Ren HG, Zhao L, Wang Z, et al.Compound danshen dropping pill for acute myocardial infarction in the rat myocardial cell apoptosis and apoptosis related proteins. Chinese journal of hardening of the arteries, 12(2013) 1084-1088.

[5] Seema P,Frederick K,Ravindra G, et al. Percutaneous Coronary Intervention Versus Optimal Medical Therapy in Stable Coronary Artery Disease: A Systematic Review and Meta-Analysis of Randomized Clinical Trials. Circulation: Card iovascular Interventions, 5(2012)476-490.

[6] Liu SQ,Tefft BJ,Zhang D,et al.Cardioprotective mechanisms activated in response to myocardial ischemia. Mol Cell Biomech, 8(2011)319-323.

[7] Seung S,Jong T P,Joo Y N,et al. Early expressions of hypoxia-inducible factor 1alpha and vascular endothelial growth factor increase the neuronal plasticity of activated endogenous neural stem cells after focal cerebral ischemia. Neural Reg Res, 9(2014) 912-918. 
[8] Tafuro S, Ayuso E, Zacchigna S, et al. Inducible adeno-associated virus vectors promote functional angiogenesis in adult organisms via regulated vascular endothelial growth factor expression. Cardiovasc Res, 83(2009) 663-671.

[9] Carmeliet P, Ferreira V, Breier G, et al. Abnormal blood vessel development and lethality in embryos lacking a single VEGF allele. Nature 380(1996) 435-439.

[10] Myriam G,Inbal A, Yuval D, et al. VEGF-Induced Adult Neovascularization: Recruitment, Retention, and Role of Accessory ,Cells. 124(2006)175-178. 